\title{
Evaluation of Vascular Changes by Optical Coherence Tomography Angiography in Patients with Asymmetric
}

\section{Glaucoma}

\section{Vallejo E1*, Gómez HF², Martínez $\mathrm{LC}^{3}$ and Arias $\mathrm{S}^{4}$}

${ }^{1}$ Ophthalmologist, glaucoma Specialist, Hospital Militar. Medellin, Colombia ${ }^{2}$ Ophthalmologist, Glaucoma Specialist, Unidad médica Clínica del Country, Colombia ${ }^{3}$ Resident ophthalmology, Fundacion Oftalmologica de Santander, Colombia ${ }^{4}$ Medical Student, Universidad de los Andes, Colombia

\section{Research Article}

Volume 4 Issue 2

Received Date: May 13, 2019

Published Date: June 07, 2019

DOI: 10.23880/oajo-16000181

*Corresponding author: Erika Vallejo, Unidad medica Clinica del Country, Mesa - Carrera 71 A \# 52-70, Medellin, Colombia, Tel: +57 3017478037; Email: erika.vallejo.m@hotmail.com

\section{Abstract}

Objective: To determine differences in vascular density of macular, disc and peripapillary areas measured by optical coherence tomography angiography (OCT-A) in patients with asymmetric glaucoma.

Methods: Retrospective, observational study. Charts of patients diagnosed with asymmetric glaucoma, with reliable 24-2 Humphrey perimetry, optical coherence tomography (OCT) and optical coherence tomography angiography (OCT-A) between February and September 2017 were evaluated. The worse eye of each patient was considered the study eye while the best one was considered the control. There was a statistically significant difference between the eyes regarding the structural and functional metrics.

Results: A total of 30 clinical records of patients who met the inclusion criteria were included. The median age was 63 years (33-81), 18 women (60\%) and 12 men (40\%). The predominant ophthalmological diagnosis was primary openangle glaucoma (70\%), followed by secondary glaucoma (16\%) and chronic angle closed glaucoma (14\%). The vascular density was significantly lower in all regions (macular, disc and peripapillary) by optical coherence tomography angiography, in all the regions evaluated in the study eyes compared to the controls (Wilcoxon Test $\mathrm{p}<0,05$ ).

Conclusions: Optic coherence tomography angiography identified lower vascular density in the studied eyes with asymmetrical glaucoma; this reduction was progressive as the severity of glaucoma increased.

Keywords: Optic Coherence Tomography Angiography; Glaucoma; Visual Field 
Abbreviations: OCT-A: Optical Coherence Tomography Angiography; OCT: Optical Coherence Tomography; SSADA: Split-Spectrum AmplitudeDecorrelation Angiography; MD: Mean Deviation; OCT-SD: Optical Coherence Tomography - Spectral-Domain; RNFL: Retinal Nerve Fiber Layer; POAG: Primary Open Angle Glaucoma.

\section{Introduction}

Glaucoma is defined as a multifactorial progressive optic neuropathy that leads to visual field deterioration, the prevalence increases with age and the most frequent form of presentation is primary open angle glaucoma (POAG) [1-3].

Angiography by optical coherence tomography (OCT) arises as a tool to study glaucoma as well as many other pathologies $[4,5]$. With the OCT-Angiography modality, it is possible to differentiate the vessels density. Thanks to several protocols such as the division of the decorrelation amplitude (SSADA) that allows the acquisition of the image defined as the amount of changes in a region by comparing it sequentially with the next one, taken precisely in the same cross section to construct a map of the blood flow, using contrast of movement [6-10]. After processing the volume scans, the OCT wave spectrum is divided into many narrow bands, which then calculate the decorrelation of reflectance amplitude in a single place [114]. This allows visualizing the retinal vasculature in three-dimensional high resolution, including small vessels. This is very important in glaucoma, because recently, part of the damage of the optic nerve is attributed to vascular alterations at the capillary level [15-19]. The vessels have been evaluated mainly in three areas, the disc, the peripapillary area, and the macular area [20-22].

This study aims to evaluate the ability of optical coherence tomography angiography to evaluate the capillary density in glaucoma. We will find out if this correlates with the changes found in the visual field and with structural alterations in optical coherence tomography in patients with asymmetric glaucoma. This retrospective study enclosed patients who attended the glaucoma unit at Unidad Médica Clinica del Country in Bogotá. This study was carried out according to the guidelines of the Helsinki Declaration and data collection was started after the study was approved by the Clinica del Country Research Ethics Committee (IRB).

\section{Materials and Methods}

A cross-sectional study based on the review of medical records was developed. Patients older than 18 and younger than 80 years with asymmetric glaucoma were included. Asymmetry was defined as a cup difference greater than 0.2 determined by the clinical examination, asymmetry of the visual field having an eye with normal mean deviation (MD) and abnormal in the fellow eye, or with mild damage in one eye and moderate or severe damage in the other one, determined by modified Hodapp-Parrish-Anderson criteria, and reliable optical coherence tomography - Spectral-domain (OCT-SD) and OCT angiogram (Nidek RS-3000 Advance) [1]. We excluded patients with non-glaucomatous optic neuropathy, pre-existing retinal pathology, OCT-SD or OCT-A images with signal strength (SSI) $<6 / 10$, or unreliable visual fields: false positives, false negatives and/ or greater fixation losses to $15 \%$. The study protocol was approved by the Institutional Review Board of Hospital Militar Central and Clinica Del Country and it followed the tenets of the Declaration of Helsinki.

We looked for correlation between decreased retinal sensitivity by sectors of the visual field and location with the position of the alteration in the OCT-A to determine correlation or coherence between the affected areas of the visual field and the thickness of the retinal nerve fiber layer (RNFL), with the areas of vascular density reduction reported in the OCT-A according to the Garway-heath map [23].

Given that capillary density can be assessed in 4 vascular layers of the retina (superficial vascular network, deep vascular network, external retina and choriocapillaris), we analysed the superficial vascular network (Internal limiting membrane - 105 mcs posterior to this), since previously published studies indicate that this vascular network supplies RNFL and ganglion cell layer, which are the structures that are mainly affected in glaucoma. Taken into consideration that the OCT-A (Nidek RS-3000 Advance) used does not have a software available so far and to give an objective value of the vascular density measurement the Free ImageJ version 2015 software was used for the particle counting (pixels/area) in the disc, peripapillary and macular areas of the OCT angiography [6,24]. Disc images $(3 \times 3 \mathrm{~mm})$ macula of $(3 \times 3 \mathrm{~mm})$ and peripapillary $4.5 \times 4.5 \mathrm{~mm}$. Images of the capillary vascular density present in the edge of the 
optical disc (up to 750 mcs away) were taken and the particle counting was done on 5 photographs, on 2 different occasions, to determine if it presented the same measurement of particles, and/or if it was standardized, on the quality of its measurements under the Bland Altman criteria [18,21].

The patients who met the inclusion criteria were selected and a database was constructed in an excel spreadsheet. The best eye of each patient was assigned to the group called "control", which corresponded to the eye with the better mean deviation (MD) and the other eye of the same patient to a group called "study" in which the eye with worse MD was included. The socio-demographic variables; perimetry metrics including MD, which allowed to classify the patients in mild, moderate or severe damage according to the modified Hodapp-ParrishAnderson criteria were evaluated as well as the visual field registration according to the Garway-Heath map. Subsequently, the database was imported into the free statistical Software R version 3.4 .2 of 2017 and Real Statistics V 5.2 of 2017, for its respective processing and exploratory data analysis. Univariate and bivariate frequency distributions, measures of central tendency, dispersion and position were applied, as well as Shapiro Wilk, Wilcoxon, Mann Whitney, Exacta de Fischer and Chi square of proportions, in the inferential analysis.

For the achievement of the objectives, we evaluated whether the data obtained in the sample presented normal distribution or not by means of the Shapiro Wilk test. The comparison of the vascular density for the three regions between cases and controls was established through the Wilcoxon test. The relationship between the level of severity and regions in case or control groups was done by Fischer's Exact test. The comparison between RNFL thickness by OCT with the level of severity by OCTA, by means of the Student's T-test. The comparison of the MD of the visual field with the level of severity by OCT-A by regions, using the Mann Whitney test and finally the proportion of agreements between control and study groups for each variable was studied by the Chi-square test of proportions between the visual field zones "Garway-heath", the corresponding OCT and OCT-A regions.

\section{Results}

A total of 30 patients were diagnosed with asymmetric glaucoma the median age was 63 years (33-81), 18 women (60\%) and 12 men (40\%). When performing the distribution, data in the control and study group by OCT, all the variables studied were statistically thicker in the control eye, and the global RNFL and peripapillary area by regions were lower with a statistically significant value in the study eyes. A redistribution of the patients in mild, moderate and severe damage was carried out in 3 subgroups according to the Hodapp-Anderson-Parrish criteria modified in terms of the decibels of the mean deviation (MD) (Figure 1). In the control group, only patients with mild and moderate defects were found, while the study group had the three levels of severity.

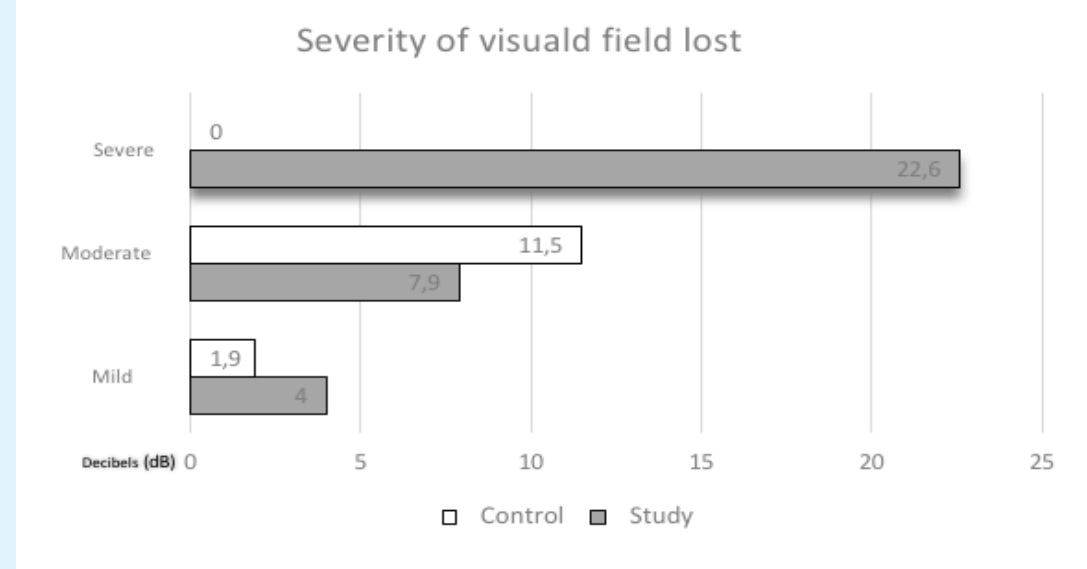

Figure 1: Average MD by the level of severity according to Hodapp-Anderson-Parrish criteria modified in the control and study groups. 
The superficial vascular network, had statistically lower vascular density in all the evaluated regions in the study eyes, compared to the controls (Wilcoxon Test $p$
$<0.05)$, results were statistically significant for all regions (Figure 2).

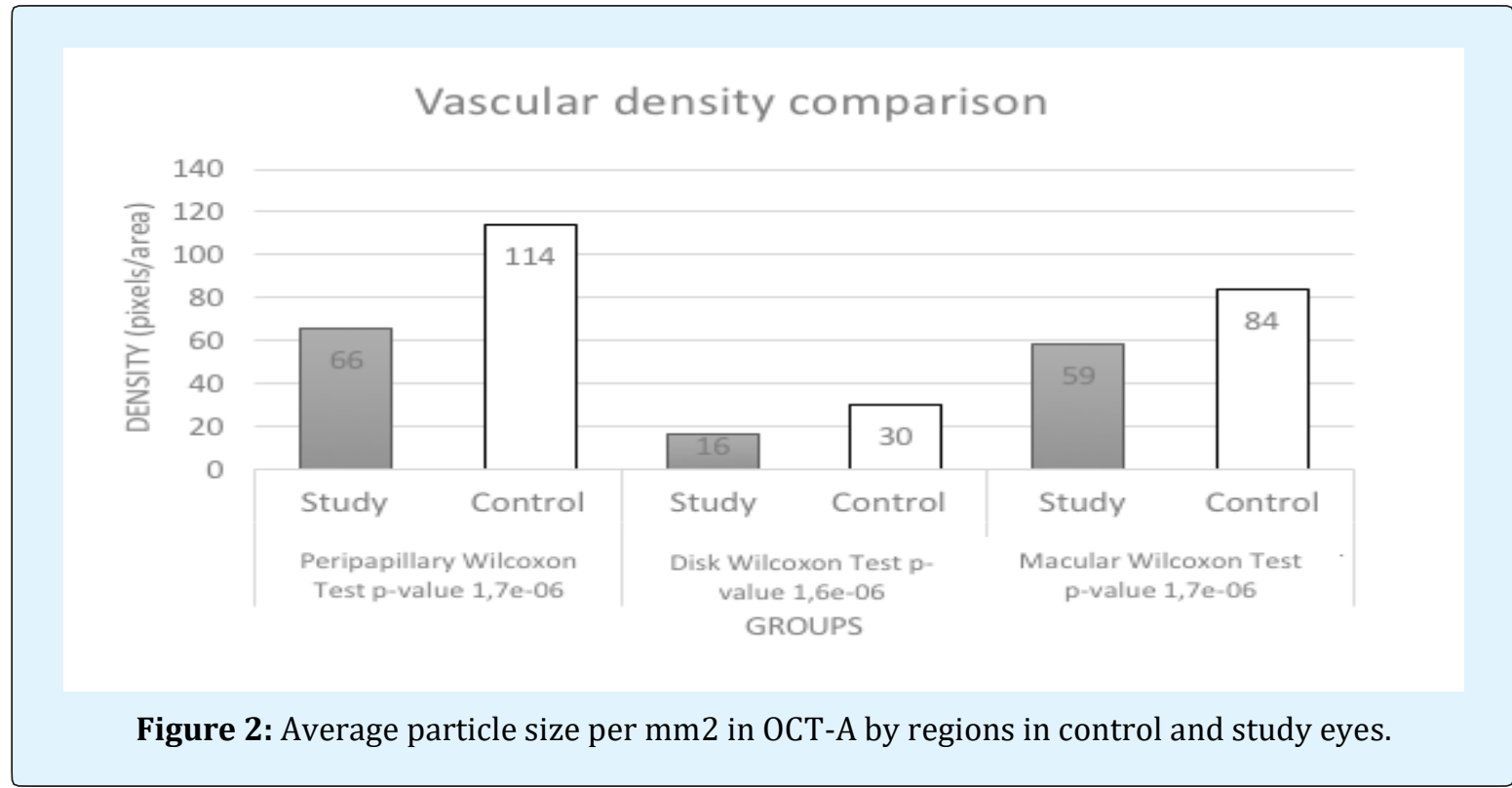

To evaluate the amount of agreement between the "Garway-heath" visual field areas described above with the corresponding regions for RNFL and OCT angiography. We found an agreement was reached for all the variables compared by Chi-squared proportions (test-p value $<0.05$ ), except for zone 1 with the temporal region of the OCT, zone 2 with the upper peripapillary region and upper macular region in OCT-A (Figures 3-5).

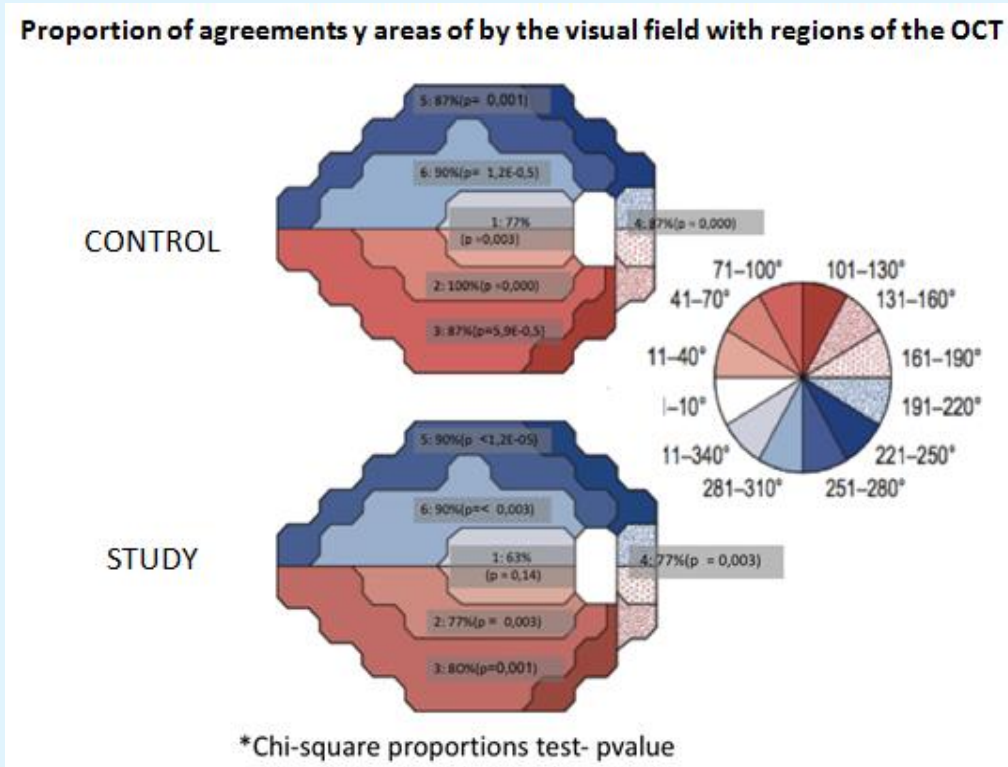

Figure 3: Agreements of proportions of visual field zones, with corresponding OCT regions. 


\section{Proportion of Agreements by Visual Field Zones with Peripapillary oct Angiography}

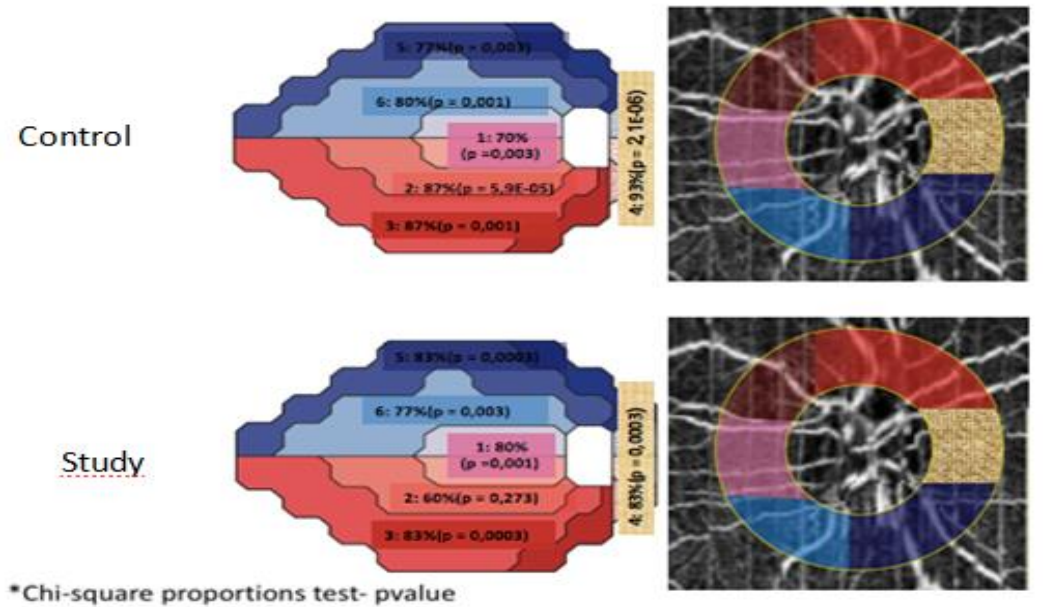

Figure 4: Agreements of proportions of visual field zones, with the corresponding peripapillary region of OCT-A.

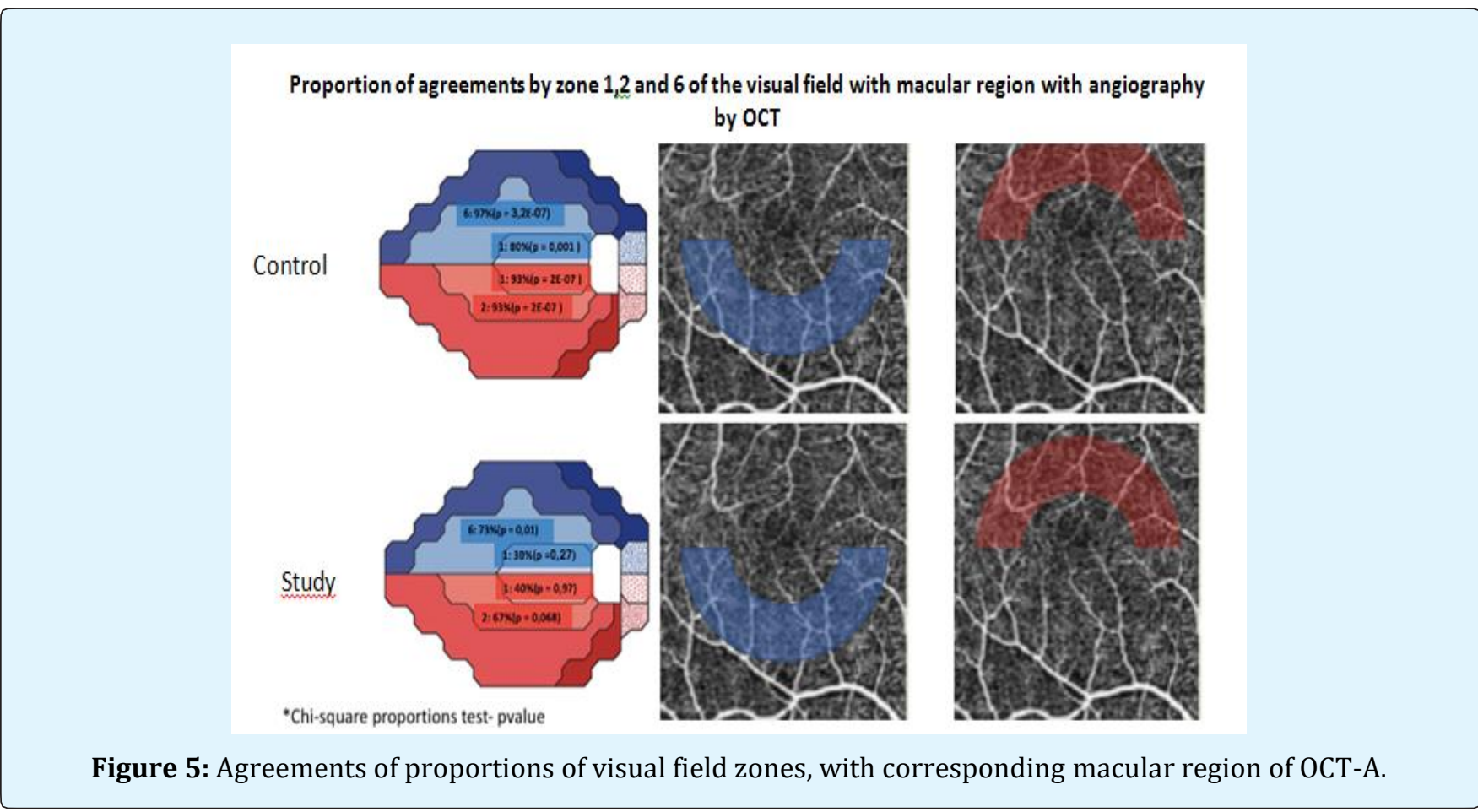

To evaluate the severity of capillary density reduction in OCT-A, according to the particle count, a distribution was made in mild, moderate and severe, for all the regions in the two groups (Figure 6). The Student's T-test was used to compare both groups according to the level of reduction of capillary density severity by OCT angiography, finding that the difference in thickness was about 30 (61-92) microns in the mild cases between the control and study eyes for the 3 regions; the study eyes being the ones with thinner values (Figure 7). However, 
this difference was not maintained when the severity stage was moderate (Figure 8), since the thickness was approximately 15 microns higher in the control eyes than in those in the peripapillary region and the disc, but the difference remains statistically significant for these two regions. Although a difference persisted in RNFL thickness at the macular level, this difference was less than about 10 microns and it did not present a statistically significant difference.

\section{Level of severity by OCT-A}

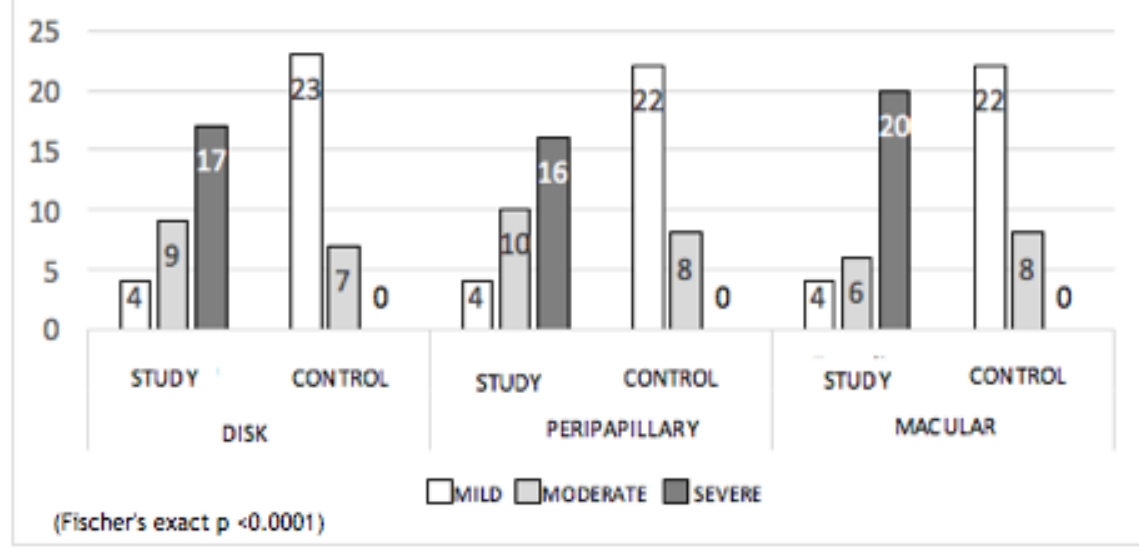

Figure 6: The number of eyes distributed by severity stage according to capillary density, for each region by groups in OCT-A.

\section{COMPARISON MILD LEVEL BY OCT-A WITH RNFL}

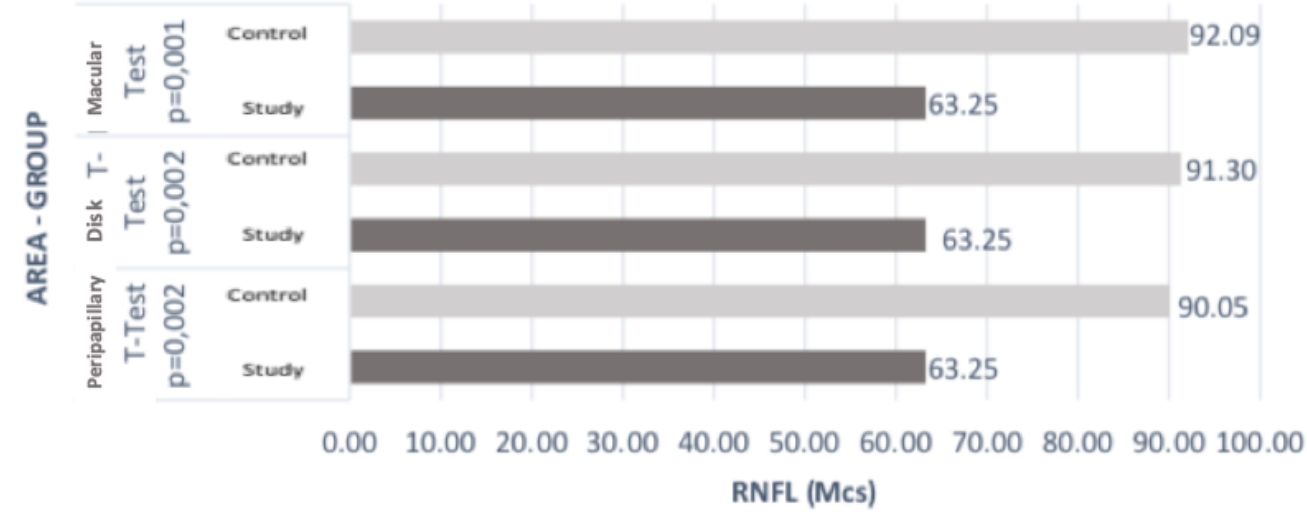

Figure 7: The average thickness of RNFL for the three regions in the mild stage of vascular density reduction. 
COMPARISON MODERATE LEVEL BY OCT-A WITH

RNFL

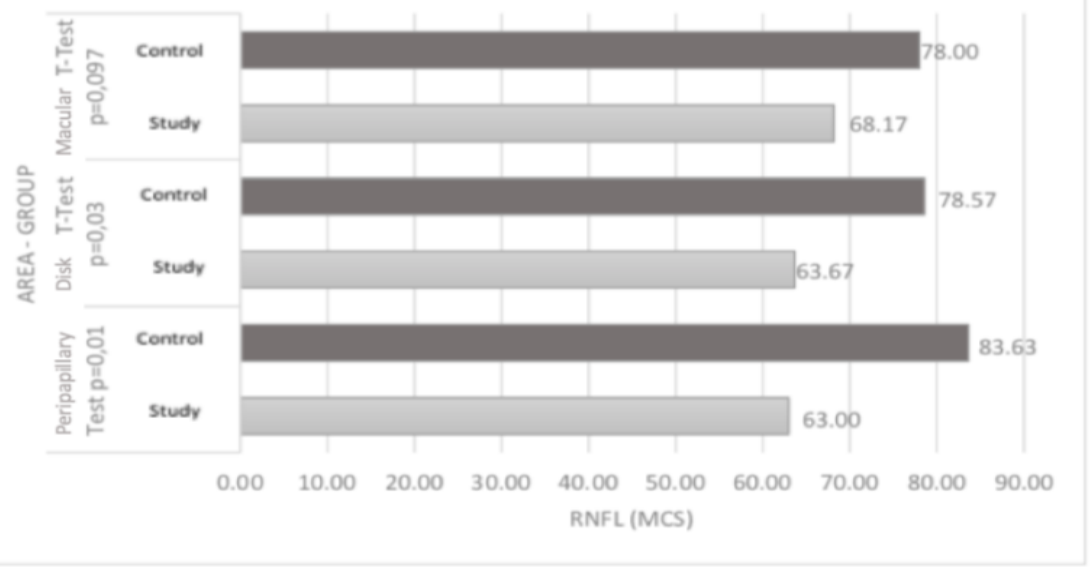

Figure 8: The average thickness of RNFL for the three regions in the moderate stage of vascular density reduction.

The groups, we subdivided according to the stages of severity given by the particle counting (pixels/area) OCT$A$, and for each group, the MD was calculated in both control eyes and study eyes by regions. We found a lower MD in all regions for the mild and moderate stages according to vascular density Mann Whitney test with a statistically significant difference (mild stage: macular $\mathrm{p}=$ 0.01 , disk $\mathrm{p}=0.02$, peripapillary $\mathrm{p}=0.02$ ) (Figure 9), (moderate stage: disk $\mathrm{p}=0.04$, peripapillary $\mathrm{p}=0.004$ ), except for the macular region in moderate stage in which there was also a difference in the control group, but this was not significant (macula $p=0.053$ ) (Figure 10). In the severe stage by OCT-A, only the MD average in the study group could be analyzed, because no patients were present in this stage in the control group.

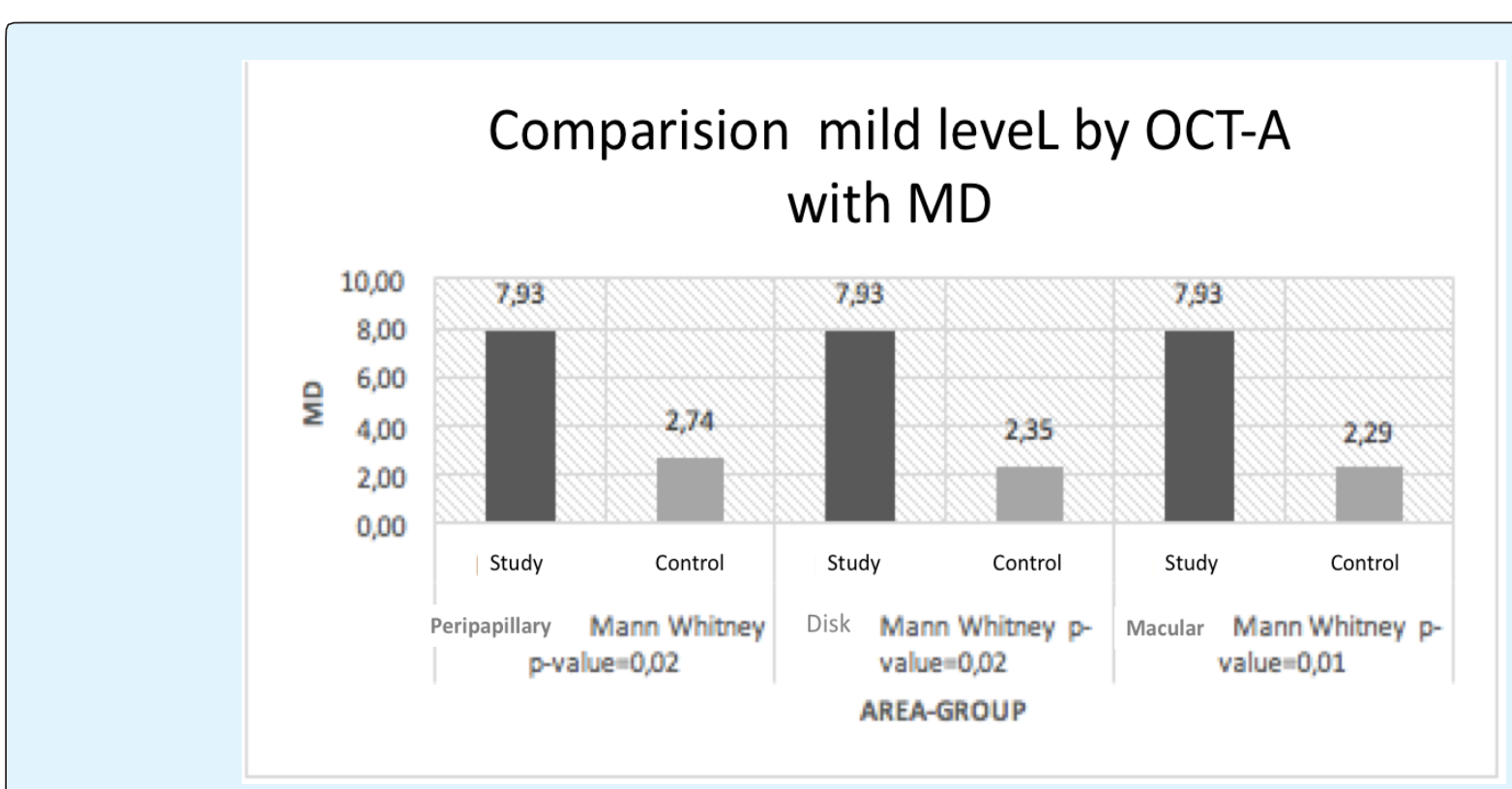

Figure 9: Average MD for the three regions in the mild stage of vascular density reduction. 


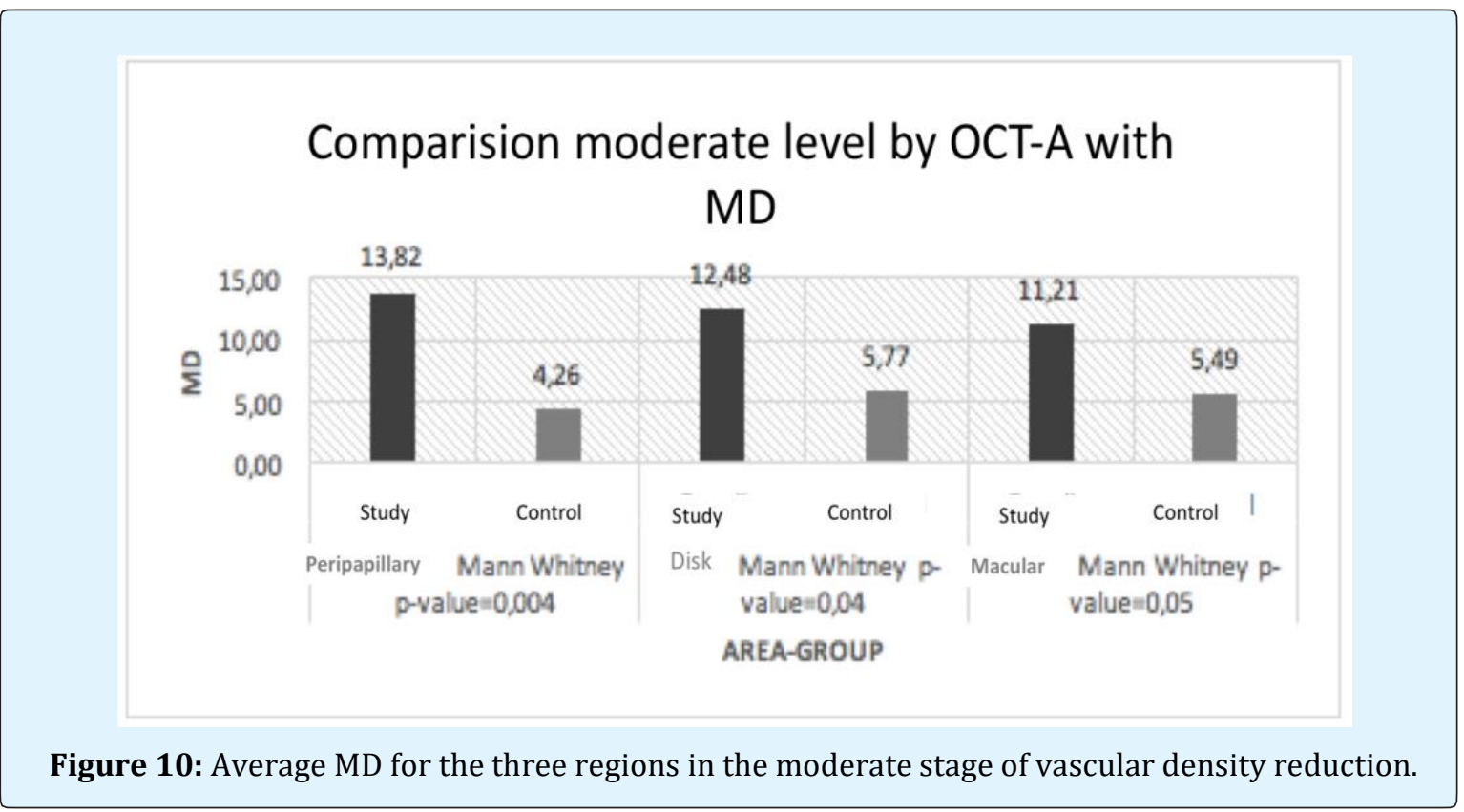

\section{Discussion}

Glaucoma is a chronic and progressive optic neuropathy, that it is not detected on time may lead to visual disability, and irreversible blindness. In order to evaluate the diagnosis and progression of the disease, different tests such as perimetry and OCT are been used in the current clinical practice $[5,6,8,10]$. However, it is still a disease that generates concern, and new diagnostic technologies are constantly being developed that allow earlier detection and better detection of progression. OCT-A is a new tool that allows us to evaluate the vascular density in different regions and layers of the retina that is being standardized for the diagnosis of multiple retinal pathologies and glaucoma.

A vascular component in the pathogenesis of glaucoma has been proposed and therefore multiple tools have been used to determine blood flow measurements. However, because multiple limitations, these tests are not used routinely in the clinical practice; the Doppler flowmetry and ocular blood analyzer have great variability, fluorescein angiography is invasive and requires long imaging times $[6,10]$. This has led to the postulation of OCT-A as a good tool to determine capillary density, although it should be remembered that it does not directly measure blood flow $[3,4,6,7]$. The difference in MD and the RNFL thickness was statistically significant between the control and study eyes. As expected, the MD was worse in the study group ( -15.3 vs. $-3.6 \mathrm{~dB}$ ) and the RNFL thicker in the control group (87.5 vs. 61.5 microns).

As previously reported by other authors Liang Liu et al., Yarmohammadi et al., Akagi et al., Rao et al., we found a lower capillary density in eyes with advanced glaucoma, when compared with the control group [20,25-27]. This density was lower in the 3 regions (disc, parapapillary and macula) as MD was worse, as reported in the study by Yarmohammadi et al. and Zhu et al. [28,29]. We also found that capillary reduction increases as the RNFL gets thinner. Another important correlation is the percentage of agreement between the different areas of the visual field and the corresponding areas of vessel reduction in OCT-A in both groups with high statistically significance. This could indicate that the evaluation of the vasculature can serve as an additional parameter in the diagnosis and follow-up of the progression in glaucoma, since not only there is a reduction of the capillaries, but also, this reduction increase with the glaucoma severity. Could also be used as a surrogate in the patient with unreliable perimetry or unable to perform it.

The reduction of vascular density has been reported to have greater diagnostic value in the peripapillary region, than in the other two regions for the evaluation of glaucoma [21]. However, in our study, the reduction was similar in the disc and peripapillary regions, but not for the macular region. 
Study limitations are related to the fact that we do not have standardized software for the evaluation of capillary density so we explored the Free ImageJ version 2015 software for the particle counting (pixels/area). In OCT-A the motion artifacts can generate hyper-reflective areas that could be interpreted as a capillary density increase in particle analysis by Image-J. In our study, we did not have into consideration the intraocular pressure of the patients or the use of glaucoma medications which can influence the measurements [30]. The stratification by level of severity according to the reduction of vascular density by subgroups was made according to the behavior of the data in our patients; this means they cannot be extrapolated to other populations.

Even though some studies have found that the reduction of vascular density could precede alterations of the visual field, vessels density reduction could be seen in ocular hypertensive patients and preperimetric glaucoma, with normal RNFL thicknesses, we did not evaluate this phenomena, by the characteristics of the study $[19,22,28,31,32]$.

\section{Conclusion}

This is the first study to evaluate vascular density at the disc, peripapillary and macular areas within patients with asymmetric glaucoma using OCT (Nidek SD 3000 Advance). The evaluation of vascular density can serve as an indicator of severity in glaucoma, since our patients with more advanced glaucoma presented a greater reduction in vascular density than eyes with early glaucoma. In addition, we found a high concordance between visual field, RNFL and vascular density values. OCT angiography is an additional tool in the diagnosis and follow-up of glaucoma. Prospective longitudinal studies with larger samples are needed and normative bases will help to standardize the vascular density.

\section{References}

1. Stamper R, Lieberman M, Michael D (2009) BeckerShaffer's Diagnosis and Therapy of the Glaucomas. $8^{\text {th }}$ (Edn.), Mosby, pp: 239-252.

2. Shaarawy T, Sherwood M (2009) Glaucoma. $2^{\text {th }}$ (Edn.), Elsevier, pp: 57-80.

3. Douglas JR, Sayoko EM, Shields B, Allingham R, Freedman SF, et al. (2010) Textbook of Glaucoma. $6^{\text {th }}$ (Edn.), Wolters Kluwer pp: 177-180.
4. De Carlo TE, Romano A, Waheed NK, Duker JS (2015) A review of optical coherence tomography angiography (OCTA). Int J Retina Vitreous 1: 5.

5. Spaide RF, Fujimoto JG, Waheed NK, Sadda SR, Staurenghi G (2018) Optical coherence tomography angiography, Prog Retin Eye Res 64: 1-55.

6. Chalam KV, Sambhav K (2016) Optical coherence tomography Angiography in retinal diseases. J Ophthalmic Vis Res 11(1): 84-92.

7. Lasave AF (2016) Interpretación actual de la tomografía de coherencia óptica en el polo posterior. Arch Soc Esp Oftalmol 91(1): 3-9.

8. Suh MH, Zangwill LM, Manalastas PIC, Belghith A, Yarmohammadi A, et al. (2016) Optical Coherence Tomography Angiography Vessel Density in Glaucomatous Eyes with Focal Lamina Cribrosa Defects. Ophthalmology 123(11): 2309-2317.

9. Gao SS, Jia Y, Zhang M, Su JP, Liu G, et al. (2016) Optical Coherence Tomography Angiography. Invest Ophthalmol Vis Sci 57(9): 27-36.

10. Coscas G, Lupidi M, Coscas F (2016) Heidelberg Spectralis Optical Coherence Tomography Angiography: Technical Aspects. Dev Ophthalmol 56: 1-5.

11. Wyle A, Wyle E (2016) Optical coherence angiography. Analysis Medicine 95: 41-51.

12. Jia Y, Bailey ST, Hwang TS, McClintic SM, Gao SS, et al. (2015) Quantitative optical coherence tomography angiography of vascular abnormalities in the living human eye. Proc Natl Acad Sci USA 112(18): 23952402.

13. Hee Suh M, Zangwill LM, Manalastas PC, Belghith A, Yarmohammadi A, et al. (2016) Deep Retinal Layer Microvasculature Dropout Detected by the Optical Coherence Tomography Angiography in Glaucoma. Ophthalmology 123(12): 2509-2518.

14. Kashani AH, Chen CL, Gahm JK, Zheng F, Richter, GM, et al. (2017) Optical coherence tomography angiography: A comprehensive review of current methods and clinical applications. Progress in Retinal and Eye Research 60: 66-100. 
15. Fan N, Wang P, Tang L, Liu X (2015) Ocular Blood Flow and Normal Tension Glaucoma. Biomed Res Int pp: 1-7.

16. Caprioli J, Coleman AL (2010) Blood Pressure, Perfusion Pressure, and Glaucoma. Am J Ophthalmol 149(5): 704-712.

17. Petrig BL, Riva CE, Hayreh SS (1999) Laser Doppler flowmetry and optic nerve head blood flow. Am J Ophthalmol 127(4): 413-425.

18. Mammo Z, Heisler M, Balaratnasingam C, Lee $S, Y u$ DY, et al. (2016) Quantitative Optical Coherence Tomography Angiography of Radial Peripapillary Capillaries in Glaucoma, Glaucoma Suspect, and Normal Eyes. Am J Ophthalmol 170: 41-49.

19. Akil H, Huang AS, Francis BA, Sadda SR, Chopra V (2017) Retinal vessel density from optical coherence tomography angiography to differentiate early glaucoma, pre-perimetric glaucoma and normal eyes. PLoS One 12(2): e0170476.

20. Rao HL, Pradhan ZS, Weinreb RN, Reddy HB, Riyazuddin M, et al. (2016) Regional Comparisons of Optical Coherence Tomography Angiography Vessel Density in Primary Open-Angle Glaucoma. Am J Ophthalmol 171: 75-83.

21. Hollo G (2016) Intrasession and Between-Visit Variability of Sector Peripapillary Angioflow Vessel Density Values Measured with the Angiovue Optical Coherence Tomograph in Different Retinal Layers in Ocular Hypertension and Glaucoma. PLoS One 11(8): e0161631.

22. Scripsema NK, Garcia PM, Bavier RD, Chui TYP, Krawitz BD, et al. (2016) Optical Coherence Tomography Angiography Analysis of Perfused Peripapillary Capillaries in Primary Open-Angle Glaucoma and Normal-Tension Glaucoma. Invest Ophthalmol Vis Sci 57(9): 611-620.

23. Garway-Heath DF, Poinoosawmy D, Fitzke FW, Hitchings RA (2000) Mapping the Visual Field to the Optic Disc in Normal Tension Glaucoma Eyes. Ophthalmology 107(10): 1809-1815.

24. Yarmohammadi A, Zangwill LM, Diniz-Filho A, Saunders LJ, Hee Suh M, et al. (2017) Peripapillary and Macular Vessel Density in Patients with Glaucoma and Single-Hemifield Visual Field Defect. Ophthalmology 124(5): 709-719.

25. Liu L, Jia Y, Takusagawa HL, Pechauer AD, Edmunds B, et al. (2015) Optical Coherence Tomography Angiography of the Peripapillary Retina in Glaucoma. JAMA Ophthalmol 133(9): 1045-1052.

26. Yarmohammadi A, Zangwill LM, Diniz-Filho A, Suh MH, Manalastas PI, Fatehee N, et al. (2016) Optical Coherence Tomography Angiography Vessel Density in Healthy, Glaucoma Suspect, and Glaucoma Eyes. Investig Opthalmology Vis Sci 57(9): 451-459.

27. Akagi T, Iida $Y$, Nakanishi H, Terada N, Morooka S, Yamada H, et al. (2016) Microvascular Density in Glaucomatous Eyes With Hemifield Visual Field Defects: An Optical Coherence Tomography Angiography Study. Am J Ophthalmol 168: 237-249.

28. Yarmohammadi A, Zangwill LM, Diniz-Filho A, Suh MH, Yousefi S, Saunders LJ, et al. (2016) Relationship between Optical Coherence Tomography Angiography Vessel Density and Severity of Visual Field Loss in Glaucoma. Ophthalmology 123(12): 2498-2508.

29. Zhu L, Zong Y, Yu J, Jiang C, He Y, et al. (2018) Reduced Retinal Vessel Density in Primary Angle Closure Glaucoma: A Quantitative Study Using Optical Coherence Tomography Angiography. J Glaucoma 27(4): 322-327.

30. Chihara E, Dimitrova G, Chihara T (2018) Increase in the OCT angiographic peripapillary vessel density by ROCK inhibitor ripasudil instillation: a comparison with brimonidine. Graefes Arch Clin Exp Ophthalmol.: 256(7): 1257-1264.

31. Kumar RS, Anegondi N, Chandapura RS, Sudhakaran S, Kadambi SV, et al. (2016) Discriminant Function of Optical Coherence Tomography Angiography to Determine Disease Severity in Glaucoma. Invest Ophthalmol Vis Sci 57(14): 6079-6088.

32. Mansouri K (2016) Optical coherence tomography angiography and glaucoma: searching for the missing link. Expert Rev Med Devices 13(10): 879-880. 